\title{
Advanced Maintenance Simulation by Means of Hand-Based Haptic Interfaces
}

\author{
Michele Nappi, Luca Paolino, Stefano Ricciardi, Monica Sebillo, \\ and Giuliana Vitiello \\ Dipartimento di Matematica e Informatica, Università degli Studi di Salerno, \\ 20186, Fisciano (SA), Italy
}

\begin{abstract}
Aerospace industry has been involved in virtual simulation for design and testing since the birth of virtual reality. Today this industry is showing a growing interest in the development of haptic-based maintenance training applications, which represent the most advanced way to simulate maintenance and repair tasks within a virtual environment by means of a visual-haptic approach. The goal is to allow the trainee to experiment the service procedures not only as a workflow reproduced at a visual level but also in terms of the kinaesthetic feedback involved with the manipulation of tools and components. This study, conducted in collaboration with aerospace industry specialists, is aimed to the development of an immersive virtual capable of immerging the trainees into a virtual environment where mechanics and technicians can perform maintenance simulation or training tasks by directly manipulating 3D virtual models of aircraft parts while perceiving force feedback through the haptic interface. The proposed system is based on ViRstperson, a virtual reality engine under development at the Italian Center for Aerospace Research (CIRA) to support engineering and technical activities such as design-time maintenance procedure validation, and maintenance training. This engine has been extended to support haptic-based interaction, enabling a more complete level of interaction, also in terms of impedance control, and thus fostering the development of haptic knowledge in the user. The user's "sense of touch" within the immersive virtual environment is simulated through an Immersion CyberForce ${ }^{\circledR}$ hand-based force-feedback device. Preliminary testing of the proposed system seems encouraging.
\end{abstract}

Keywords: Haptics, Virtual Reality, Multimodal Interfaces.

\section{Introduction}

In the last years the industrial world has been increasingly adopting computer-aided solutions for design-for-maintainability and maintenance training tasks with the goal to reduce development costs and to shorten time, and to improve product and service quality. Indeed, when technical systems are not designed and realized taking into account future maintenance operations, they imply high times and costs even for a simple replacement intervention [1]. For these reasons maintainability has to be considered a design added value and a competitive factor [2]. The term maintainability represents the synthesis of several characteristics that are settled on during the design 
phase. In fact, in this phase the critical areas to carry out maintenance tasks can be identified, the necessity of using instruments for failures diagnosis can be highlighted, and, at last, special equipment for maintenance can be devised and developed. For these reason it is clear that the design for maintainability has a positive effect on the operational costs that the user will face using the system. This can probably imply a bigger purchase price, but the final objective is to minimize the overall life cycle cost [3]. Carrying out simulations of maintenance activities within a virtual environment gives a person the ability to directly interact with $3 \mathrm{D}$ virtual models for maintenance purposes. Engineers can employ it to evaluate aspects of human-centered design for maintainability (accessibility, reachability, tool usability, part mount/dismountability). With respect to well-established techniques based on the intermediation of virtual characters, the first-person approach offers a more direct, intuitive control over the interaction activity, thus speeding up the maintenance checks, along with the opportunity to find out better design solutions from the maintainability point of view. Furthermore, maintenance operators (mechanics, technicians) can be trained within a highly interactive, realistic virtual reality simulator, thus combining advantages of a safe training environment with the value of the "learning by doing" practice.

To this regard, ViRstperson is a virtual reality software system developed at the Italian Center for Aerospace Research (CIRA) Virtual Reality Laboratory for carrying out digital maintenance simulations by a first-person approach $[4,5]$. It is aimed at supporting engineering and technical activities such as design-time maintenance procedure validation and maintenance training. Techniques employed for improving the realism of the interactive experience include advanced lighting and shadowing to improve the user's spatial awareness within the virtual environment and a complete dynamics simulation facility which rules the interaction of all bodies within the environment, including sensor-attached anthropomorphic parts (e.g. a digital glove) [6, 7]. Both a desktop and an immersive approach can be employed by means of display systems such as auto-stereoscopic screens, mono/stereoscopic projection systems, and head-mounted displays. Computer Based Training systems created to simulate machine assembly maintenance are typically operated by means of ordinary humancomputer interfaces (keyboard, mouse, etc), but this usually results in systems that are far from the real procedures, and therefore not effective in terms of training.

This study presents an extension of ViRstperson to address the simulation of the haptic feedbacks provided during the manipulation of components and equipments involved during service or maintenance operations for aerospace industry. The usersystem interaction is accomplished by means of commercially-available head/hand trackers, digital gloves (CyberGlove ${ }^{\circledR}$ ) and Immersion CyberForce ${ }^{\circledR}$ force-feedback system [8]. The goal is to allow techinical people to be trained to maintenance tasks, enabling them to perceive the physical characteristics of the simulated environment in a realistic way, thus improving their overall knowledge of the procedures as well as their efficiency.

This paper is organized as follows. In section 2. the relationships between haptic interfaces and system dependability are briefly exposed. In section 3. the proposed approach to visual-haptic maintenance simulation is presented in detail, while in section 4. the first experiences with the training simulator are exposed and discussed. The paper concludes in section 5 . 


\section{Haptic Interfaces and System Dependability}

When designing haptic systems, two different aspects may cause difficulties. One is related to the haptic device's functional requirements, the other is related to the associated user interface (user interface requirements). Haptic devices should be designed to guarantee dexterity (no motion constraints causing uneasiness in the extended use), human safety and portability (robust, dependable, easy to fit and adjust and be selfcontained with wireless communications) $[9,10]$. However, an effective use of those devices may be strongly affected by the lack of dependability in the user interface, which is the second important property haptic system designers should strive for.

Dependability of a system is commonly referred to its Reliability, its Availability and its Maintainability (RAM). However, when focusing on the user interface, there is no common agreement on what aspects of user-system interaction are related to a satisfactory RAM level of the whole system. According to [11], a dependable user interface should support speedy and accurate completion of user tasks. One of the major causes for failure with respect to accuracy and speed specifications is in fact human error. If a user makes an error while performing a task, this will propagate to the subsequent steps and result in incorrect task completion. Therefore, human computer interaction researchers strongly recommend that interface designers put special focus on error prevention, besides error recovery activities [12, 13]. Designers should investigate the most common errors users of the target domain may perform on the interface, and the most common causes for those errors, in order to understand how to prevent them, and consequently reduce the risk of mission failures in the underlying system. When dealing with haptic systems, interface dependability may become a crucial issue, especially when:

a. life-critical systems are to be manipulated, e.g., in medical and in military domains;

b. costly remote control operations are to be performed, such as product manufacturing, automotive or aerospace engineering.

Several cognitive studies on the human perceptual system related to the sense of touch, have tried to identify what human factors should be considered to connect the physical hand movements with a virtual world, as displayed, e.g., by visual interfaces or by more complex immersive environments $[14,15,16]$. Some of those studies focus on the tactile feedback, dealing with devices able to transmit to user's hand nerve endings heat, pressure or texture sensations. Other studies have instead considered the area of force feedback, usually adopted for devices such as robotic manipulators which interact with user's kinesthetic system (muscles, tendons and joint), applying forces as if they were generated by the virtual environment the user is operating in. The latter category also encompasses force feedback hand-based devices like the Immersion CyberForce ${ }^{\circledR}$ which has been adopted as haptic interface for the proposed simulator. Some authors already point out the importance of physical interaction issues for an accurate design of mechanism, sensors, actuators and control architecture in haptic systems [17]. They also observe that when operating with haptic devices, cognitive and physical interactions tightly depend on each other: "physical interaction can help in setting rules for cognitive evaluations of the environment, while cognitive aspects may improve the physical interaction by setting suitable control interaction 
parameters." If, for example, haptic interaction is used to explore the shape or the consistency of an object cognitive-based inference rules can be considered for compliance control of the device which physically interacts with humans during manipulation tasks. Conversely, the force/tactile feedback is used to convey to the user a comprehension of the physical characteristics of the virtual environment, and of the manipulated objects.

\section{Haptic-Based Maintenance: The Simulation Engine}

When a human has to perform a real time simulation based on the direct manual interaction approach, some parameters determine the usability of the VR system and, consequently, the reliability of the tests. Some aspects, such as the care in the simulation modelling and the visual quality, have an effect on how much realistic the user perceives the behaviour of the simulated environment, for example in terms of the reaction of the objects to the direct interaction. Even if with a more soft threshold, the parameter related to the realism influences the information obtainable from the simulation. With regard to the realism of a VR simulation, determinant aspects are basically three: the visual realism, the behaviour of the simulated world and the conveying of the sensations. In order to improve the visual realism of a virtual environment and to raise the acceptability threshold of the experience, shadows run a decisive rule. In particular, shadows are very useful to perceive the spatial relations between the objects within the scene, such as, for example, the position of the virtual hand, controlled by means of a digital glove, with regards to those objects in the scene that have to be manipulated. In order to improve the realism in the interaction the key is the correct modelling of the physical laws ruling object interaction. In this way, the virtual objects can have a reaction very similar to that one they should have in the reality, both in the direct interactions and in those deriving from the transmission of forces through collisions and/or pre-established kinematical chains.

The immersive 3D visualization system, named ViRstperson, is based on an opensource scene graph library, the Plib [18], whose main part, the SSG (Simple Scene Graph), is, in turn, based on OpenGL for real-time 3D rendering. The SSG creates and manipulates a hierarchical data structure describing the scene content in terms of nodes representing graphical objects (triangle meshes), attributes and materials, or transformations.

Aiming at developing a realistic visual and interaction virtual environment, ViRstperson has been improved with many visual and interactive functionalities, including advanced lighting, soft shadowing, stereoscopic rendering and its overall architecture is exposed in Fig. 1.

A generalizing approach to get realistic object behaviour relies on the simulation of the dynamics of objects considered as rigid bodies [19]. According to classic physic's laws, such a simulation calculates the motion of these bodies, each with assigned mass properties and shape, subjected to forces such as gravity, contacts (collisions between bodies), user-imposed, and various types of constrains. Contact forces are also treated as constraint reactions originated by the collision of two bodies. The collision detection phase, where contacts are located in, updates the world of simulated bodies with contact constraints, which subsequent simulation translates in corresponding repulsion forces. 


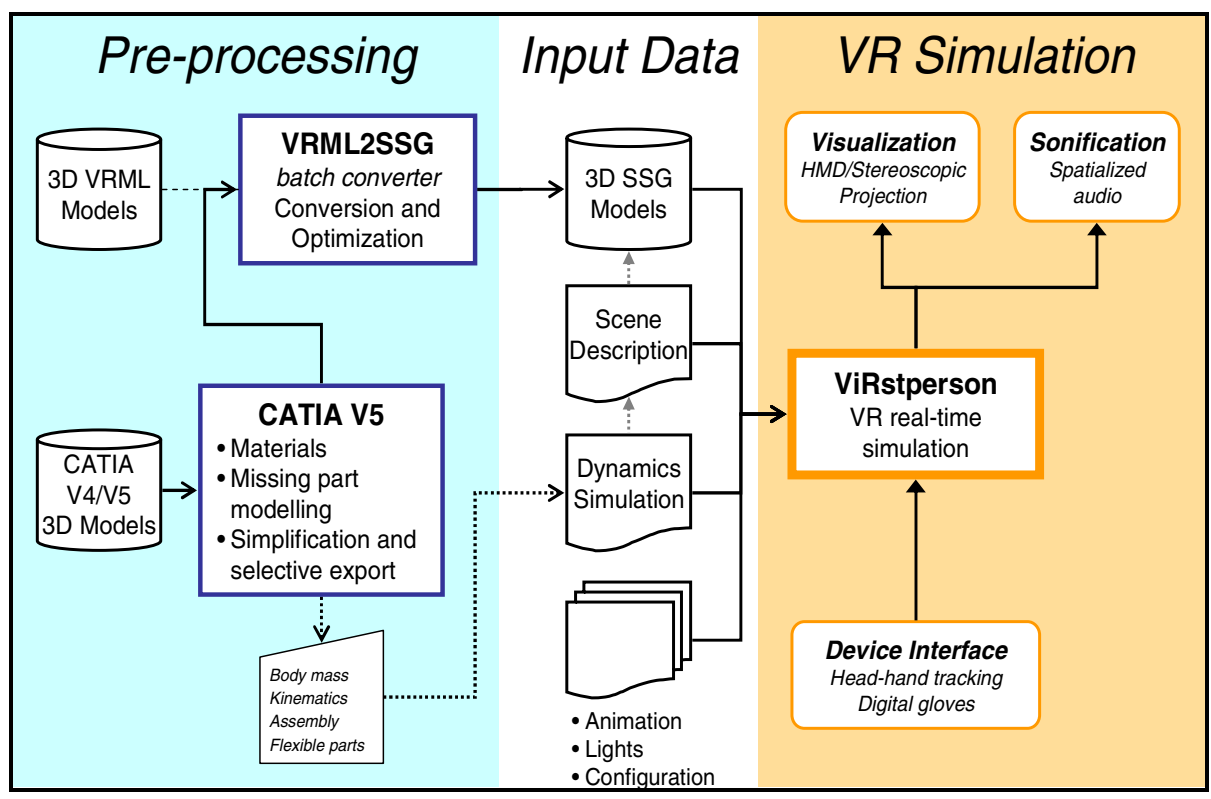

Fig. 1. A schematic view of the ViRstperson simulation engine

The Open Dynamics Engine (OpenDE) open-source library has been integrated in ViRstperson for adding dynamics simulation functionalities [20]. An OpenDE-based simulation is not strictly real-time, in the sense that it doesn't guarantee to update the system in a certain fixed time, yet it's sufficiently fast for interactive, man-in-the-loop application such as a VR simulation (Fig. 2a-2b). Performances may vary depending on the complexity of the virtual world (number of bodies and their geometric complexity, number of constraints): given a world, one can choose a combination of the solver type ("big matrix" or iterative) and the integration step (time) in order to find a suitable balance between accuracy and speed. For maximum computing efficiency on multiprocessor computers the dynamics simulation is implemented as a separate thread which shares the scene graph access with the visualization thread.

A further performance improvement is expected from the parallelization of the collision detection. In fact while the computing complexity of the simulation (integration) step is related to the sum of the number of bodies and joints, the complexity of the collision detection is related to the number of objects (which are either the "skin" of bodies or static parts of the dynamics world) and their geometry complexity (i.e. the number of triangles of their tri-meshes).

Normally when dealing with industrial, CAD-originated parts the collision detection occur among thousand of objects and some millions of triangles, whilst the number of bodies (i.e. the "movable" parts of the world) and the number of joints (the "behavioural" constraints between bodies) stay below two tens. Thus since the collision detection between pairs of objects is an inherently parallel task, a further threaded architecture will take advantage of recent multi-core processors with two or more processor per machine. 


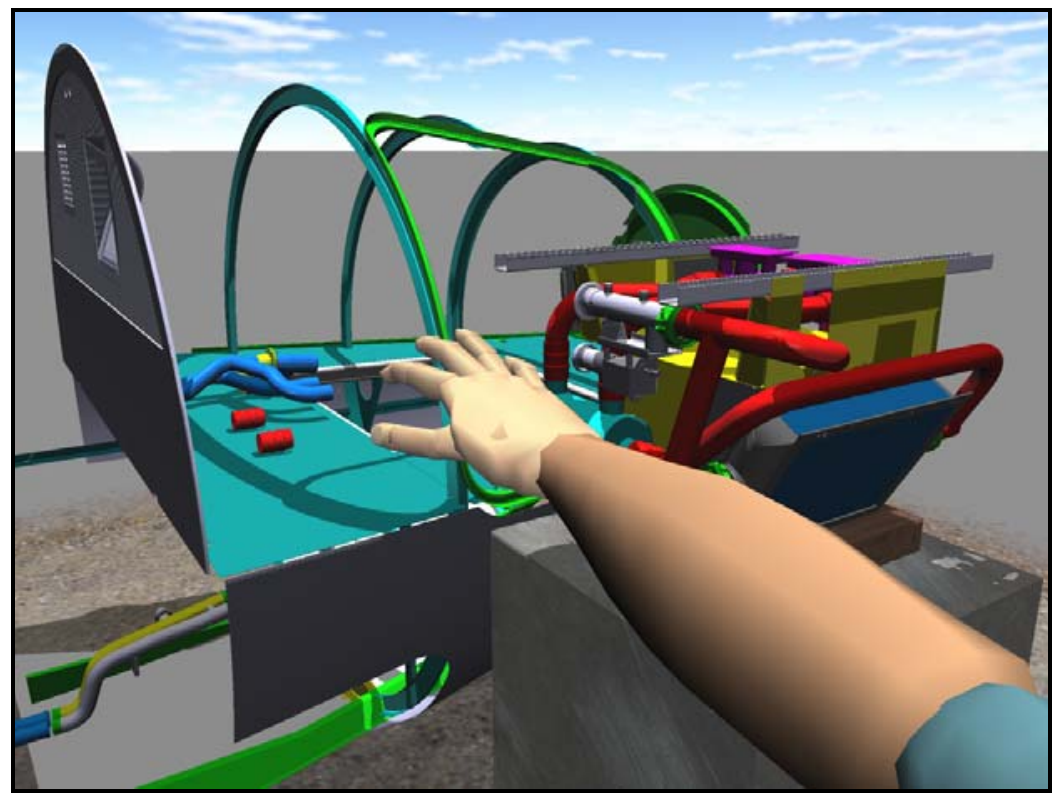

(a)

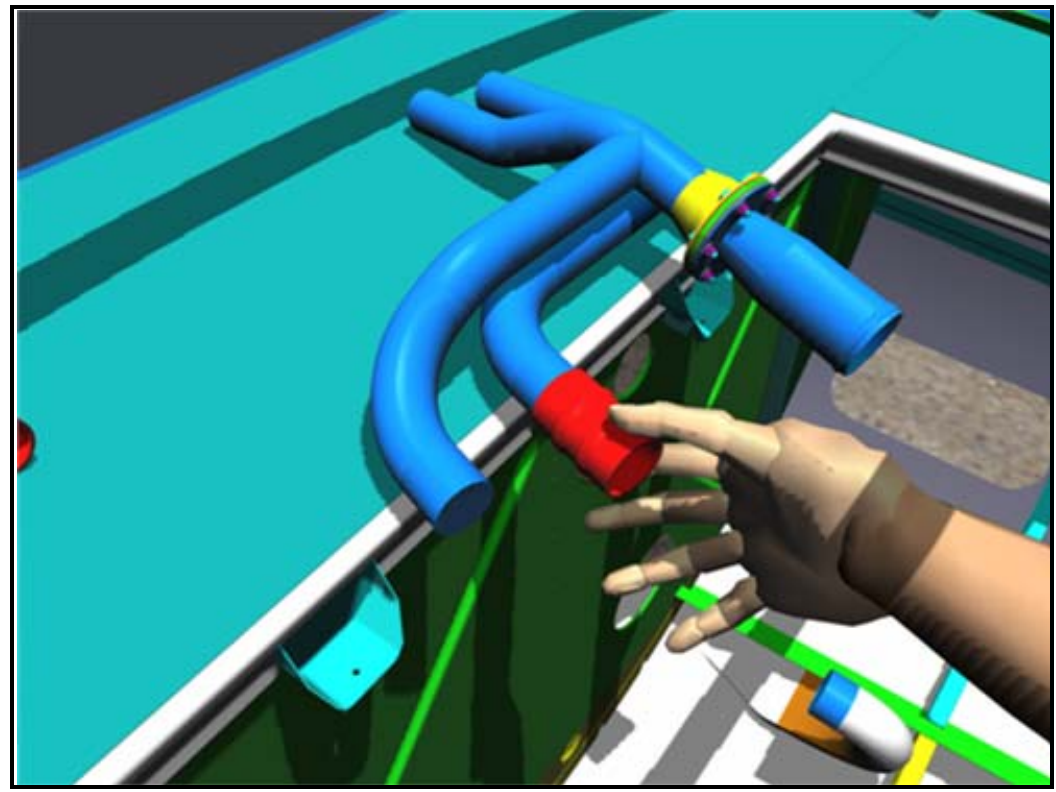

(b)

Fig. 2. Sample screens captured during direct first-person hand interaction in a virtual maintenance application using the ViRstperson VR engine 
At the end of the whole collision detection task the resulting contacts calculated by each thread would be recollected in a single array for feeding the simulation step. Thus since the collision detection between pairs of objects is an inherently parallel task, a further threaded architecture will take advantage of recent multi-core processors with two or more processor per machine. At the end of the whole collision detection task the resulting contacts calculated by each thread would be recollected in a single array for feeding the simulation step.

\subsection{Adding the Sense of Touch}

The development of a haptics-assisted virtual aircraft maintenance environment is very complex because of the difficulty to simulate realistic physical processes and due to limitations of the currently available VR devices. The research activity carried out in this field focused on particular tasks of the maintenance activity such as disassembly, accessibility and manipulability assessment of geometrically-complex mechanical systems. The virtual aircraft maintenance environment reported in this paper is focused on both main industrial maintenance fields that are design for maintainability evaluation and training of maintenance operators. During the execution of maintenance tasks the contact among virtual objects, and between hand and object, has to be as much as possible compliant to dynamic behaviour which rules the interaction between physical objects in the real world.

In this context, one of the most important sensation to be transferred to a human, is the force feedback. It allows to transfer to the user's hand the sensation to touch an object, to perceive the nature of its surfaces and/or to feel the resistance that the manipulated body oppose to manipulation because of its mass properties. This feature is particularly important in VE's for maintenance training application. In fact, in the reality an operator gets used to employ tools and parts to be maintained that have mass and stiffness and the VR system has to transmit him/her the same feeling. If these factors are not taken into account during the virtual training, such training risks to be useless or having negative impact [21].

The system proposed computes contact force rendering by making its intensity dependent on the depth of penetration of the tracked hand model into the grasped object $[22,23]$. The force calculated is felt by the operator through the haptic interface when the hand touches an object and/or parts come in contact during the simulation phase of the maintenance task. The penetration of objects into other objects in the scene is thus prevented by the combination of the collision detection system implemented in ViRstperson and the consequent force actuation by the force feedback device.

A CyberForce ${ }^{\circledR}$ force feedback system by Immersion Corporation has been utilized in order to provide the user with haptic sensations during the execution of maintenance tasks. The Cyberforce is composed by an articulated exoskeleton ending with a CyberGrasp system including a CyberGlove 22 sensors dataglove (see Fig. 3). The CyberGrasp provides the transmission of contact sensations to the operator's fingertips while the whole CyberForce simulates the weight and the inertia of the manipulated objects within its operative volume. The weight of the grasped object can be rendered by the CyberForce through the application of a force on the back of the user's hand. The operator can, therefore, estimate the accessibility of the tools and the possibility to remove components directly during the manipulation [24], since the 


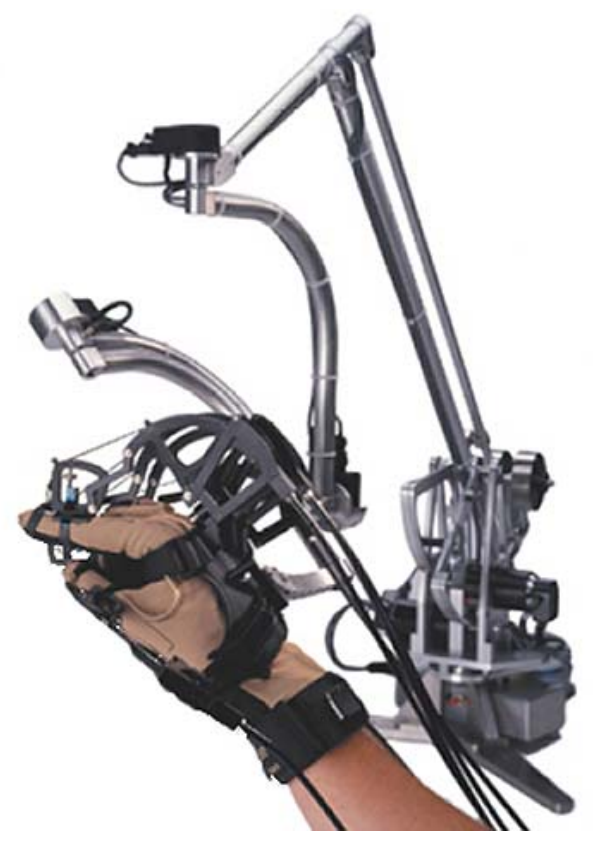

Fig. 3. The Immersion CyberForce articulated exoskeleton and the related CyberGrasp

penetration among objects gets cancelled by the reaction forces activated in relation to contacts among an object and the objects of the virtual hand [25]. Moreover the implementation of a system that allows to obtain a force feedback effect of the collisions helps the operator to notice also contacts that possibly occur outside of his field of view (e.g. contacts between elbow and objects), as long as a credible way to convey such kind of alert through the hand will be devised.

From a software point of view the integration of the CyberForce suite and ViRstperson (see Fig. 4) relies on the the product's Virtual Hand SDK for accessing device functionalities through the network [26]. On the application (client) side ViRstperson reads/writes hand-relevant data to specific shared memory segments, in order both to be general and to avoid linking with device-specific libs. External, device-specific interface programs link to the actual devices. For the force-feedback glove ViRstperson reads the sixteen $4 \times 4$ transformation matrices for the virtual hand's objects, plus the wrist position/orientation supplied by the hand tracker. It also outputs force-relevant data, which consist in one penetration depth value for each finger, plus a 3-component vector of the force vector to be actuated by the hand back force transmission arm. Then the ViRstperson CyberForce Hand Device Interface program translates such data in CyberForce-compatible force ranges for fingers and hand back actuators. The hardware configuration includes a dual 2,4 GHz Opteron based workstation equipped with a NVidia Quadro 4500 graphics board, a CyberMind Visette SXGA stereoscopic Head Mounted Display and CyberTrack hand tracking system as part of the CyberForce (see Fig. 5). The average scene load on which the system has been tested is in the range of 1 to 2 millions triangles, as the number and the geometrical complexity of many items results from the importing of CAD data. 


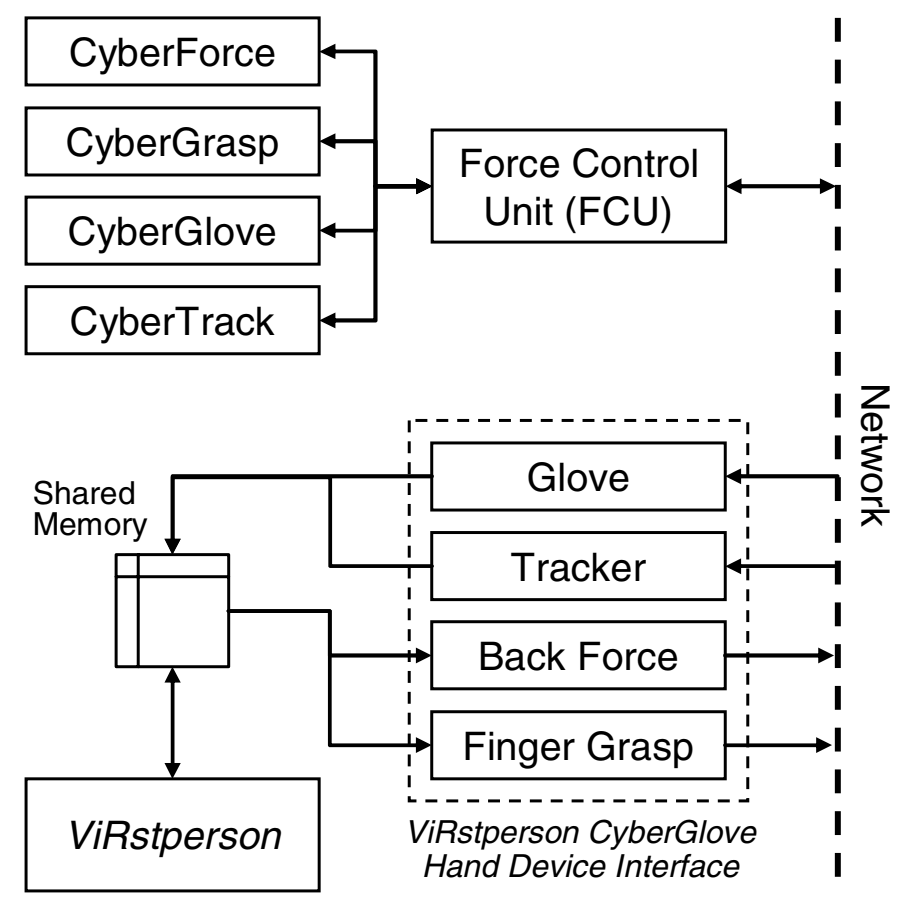

Fig. 4. Schematic view of the data channels involved in the communication between the CyberForce system and ViRstperson engine

\section{First Comments on Using the Haptic-Enabled Maintenance Simulator}

The simulation of maintenance tasks in a virtual environment heavily relies on the environment awareness gained by users through intuitive and natural interaction. The execution of virtual maintenance task through specific 3D interaction devices significantly affects the performance of the users, and contact force sensation can enhance the user presence in the virtual environment, and consequently his performance during the task execution [27, 28, 29]. Nevertheless the sensation of contact forces is not the only factor that affects human performance. The nature of interaction with virtual environment, the difference in perception levels between maintenance task execution in real world and in computer generated environment, human factors, ergonomic issues, and other considerations related to the effects of human computer interaction have to be taken into account for an objective evaluation of the developed interaction technique and to measure its effect on user performance in virtual maintenance tasks.

The development of a haptics-based interaction paradigm can contribute to the improvement of maintenance task execution, particularly when the manipulation of the objects requires a high level of perception and the execution of virtual maintenance tasks is not simply assembly-disassembly 3D models of parts. An important aspect of this application is the capability of the whole haptic device plus haptic rendering to 


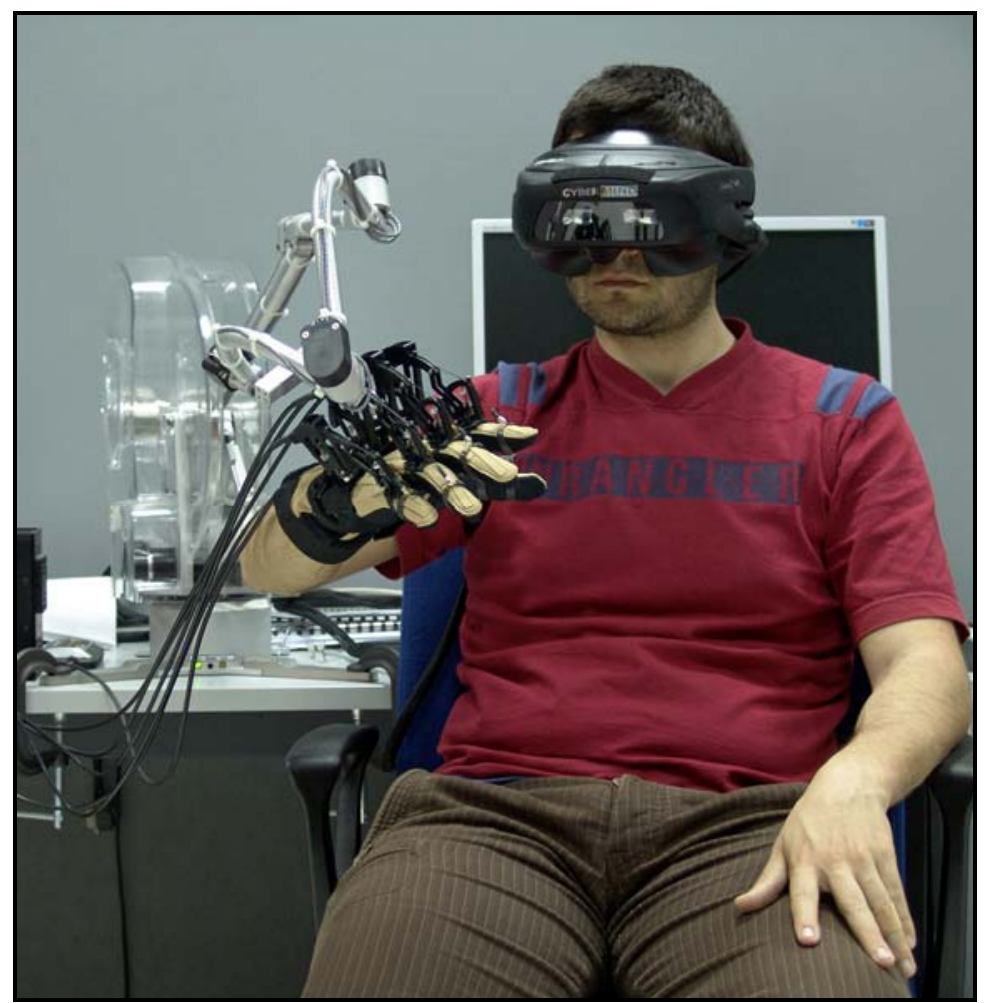

Fig. 5. Direct first-person hand interaction in the proposed virtual maintenance application using the CyberForce force feedback system

faithfully replicate the forces involved in the manipulation of technical components aboard aircraft. Indeed the mass and weight of each component as perceived by the system's user should be comparable to the real counterparts (in a range varying from hundreds of grams to several kilos). Even the operative volume can have a direct impact on the realism of the simulation if it does not allow to reach some equipments, but in this case a simple yet adequate solution could be provided by virtually shifting the haptic-device location in the virtual environment. Unfortunately, at this moment the CyberForce is one of the very few commercially available example of hand based haptic device, so its design implies some mechanical and operational limitation (Force Generation: $8.8 \mathrm{~N} \max / 6.6 \mathrm{~N} \mathrm{~min}$ ). One interesting option is the possibility to use two CyberForce arms in conjunction, to simulate two hands manipulation and coordination which could be crucial for many kind of real activities and thus indirectly doubling the forces generated during interaction. As this study is still in a early stage we can not objectively compare the "conventional" VR based training simulation to the haptics-based approach proposed.

Indeed, this will be one of the main purposes of a comprehensive evaluation study which is currently in progress, taking into consideration aspects such as usability, acceptability, level of correspondence of haptics based perception of the virtual working environment to the "sense of touch" based knowledge of the real environment. 
Another usability factor which may directly affect dependability of a haptic system is robustness. Robustness is defined as the support the system gives users to let them figure out if they have achieved their goals, and how well [30]. Considering the type of certain critical haptic interaction tasks, a sufficient degree of robustness is needed to safely and effectively achieve the initial interaction goals. Several features can be considered important to achieve robustness when dealing with a haptic interfaces. These are again related to both the haptic and the visual/audio input/output modes that the system is adopting. In particular, user's ability to evaluate the internal state of the system from its perceivable representation, and the persistence of the effect of an action on the interface (e.g., the weight of the manipulated rigid body perceived as long as it is held by the cyberglove). Also forward/backward recovery actions should be allowed as far as possible during task performance. Anyway, preliminary testing conducted on the virtual model of an avionic system performing simple to medium complexity manipulation tasks (e.g. to inspect equipments, to mount/unmount components, to replace items, etc.) with and without the haptic interface resulted in an explicit preference for the haptics-assisted modality encouraging the authors to proceed in this research.

\section{Concluding Remarks}

In this paper a framework for haptics-assisted VR based aircraft maintenance training has been presented. The system integrates a hand based force-feedback haptic device with a real-time VR simulation engine supporting realistic interaction through dynamics simulation. Although an extensive usability study has not been completed yet, the first impressions of prospective users seem to confirm the added value of haptic-based interaction compared to an exclusively visual-based training simulation. Moreover, the use of a hand-based haptic device versus a stylus-based end effector has been very favourably acknowledged by technical personnel already accustomed to VR-based training procedures, proving that for real world operations at least a force-feedback on the whole hand is required. Future work will include an extensive experimentation aimed to measure the advantages of the haptic-based approach applied to virtual training and the extension of the framework to a two-hands system which could bring even more realism to the interaction.

\section{Acknowledgements}

We gratefully acknowledge the contribution of the Italian Aerospace Research Center (CIRA) to the development of this research.

\section{References}

1. Hubka, V.: Scienza della progettazione. In: Masson Editoriale ESA, pp. 23-56 (1994)

2. Ivory, C.J., Thwaites, A., Vaughan, R.: Design for maintainability: the innovation process in long term engineering projects. In: Proc. of the Conference The future of innovation studies, Eindhoven, Netherlands (2001) 
3. Serger, J.K.: Reliability Investment and Life-Cicle Cost. IEEE Transaction on Reliability 32, 259-263 (1983)

4. Liang, J., Shaw, C., Green, M.: On temporal-spatial realism in the virtual reality environment. In: proceedings of the ACM Symposium on User Interface Software and Technology, pp. 19-25 (1991)

5. Ware, C., Rose, J.: Rotating virtual objects with real handles. ACM Transactions on CHI 6(2), 162-180 (1999)

6. Rezzonico, S., Huang, Z., Boulic, R., Magnenat Thalmann, N., Thalmann, D.: Consistent Grasping Interactions with Virtual Actors Based on the Multi-sensor Hand Model. In: Virtual Environments 1995, pp. 107-118. Springer, Wien (1995)

7. Poupyrev, I., et al.: Go-Go Interaction Technique: Non - Linear Mapping for Direct Manipulation in VR. In: ACM UIST 1996, pp. 79-80 (1996)

8. Immersion Corp 3D Interaction Hardware and Software Products, http: / /www. immersion. com/3d/

9. Biggs, S.J., Srinivasan, M.A.: Haptic Interfaces. In: Stanney, K.M. (ed.) Handbook of Virtual Environments: Design, Implementation, and Applications, pp. 93-115. Lawrence Erlbaum, Mahwah (2002)

10. Hale, K.S., Stanney, K.M.: Deriving Haptic Design Guidelines from Human Physiological, Psychophysical, and Neurological Foundations. IEEE Computer Graphics and Applications 24(2), 33-39 (2004)

11. Reeder, R.W., Maxion, R.A.: User Interface Dependability through Goal-Error Prevention. In: Proceedings of the 2005 International Conference on Dependable Systems and Networks, DSN 2005 (2005)

12. Nielsen, J.: Heuristic evaluation. In: Nielsen, J., Mack, R.L. (eds.) Usability Inspection Methods. John Wiley \& Sons, New York (1994)

13. Shneiderman, B., Plaisant, C.: Designing the User Interface: Strategies for Effective Human-Computer Interaction, vol. 4/E. Addison-Wesley, Reading (2005)

14. O’Malley, M., Goldfarb, M.: The Implications of Surface Stiffness for Size Identification and Perceived Surface Hardness in Haptic Interfaces. In: Proc. IEEE Int'l Conf. Robotics and Automation, pp. 1255-1260 (2002)

15. Sherrick, C.E., Cholewiak, R.W.: Cutaneous Sensitivity. In: Boff, K., Kaufman, L., Thomas, J. (eds.) Handbook of Perception and Human Performance v.1: Sensory Processes and Perception. John Wiley \& Sons, Chichester (1986)

16. Tan, H.Z., et al.: Human Factors for the Design of Force-Reflecting Haptic Interfaces. Am. Soc. of Mechanical Engineers DSC 55-1 (1994)

17. De Santis, A., Sicilian, B., De Luca, A., Bicchi, A.: An atlas of physical human-robot interaction. Journal of Mechanism and Machine Theory 43, 253-270 (2008)

18. Baker, S.J.: PLIB: A Suite of Portable Game Libraries, http://plib.sourceforge.net

19. Garbaya, S., Zaldivar-Colado, U.: The affect of contact force sensations on user performance in virtual assembly tasks. Journal of Virtual Reality 11(4) (October 2007)

20. Smith, R.: ODE: Open Dynamics Engine, http:/ / ode. org

21. Liang, J., Shaw, C., Green, M.: On temporal-spatial realism in the virtual reality environment. In: Proceedings of the ACM Symposium on User Interface Software and Technology, pp. 19-25 (1991)

22. Borst, C.W., Indugula, P.: Realistic virtual grasping. In: Proceedings IEEE virtual reality conference (VR 2005). Bonn, Germany, vol. 320, pp. 91-98 (2005)

23. Borst, C.W., Indugula, A.P.: A spring model for whole-hand virtual grasping. Presence Teleoperators Virtual Environ 15(1), 47-61 (2006) 
24. Chryssolouris, G., Mavrikios, D., Fragos, D., Karabatsou, V.: A virtual reality-based experimentation environment for the verification of human-related factors in assembly processes. Robot Comput. Integr. Manuf. 16(4), 267-276 (2000)

25. Colgate, J.E., Grafing, P.E., Stanley, M.C., Schenkel, G.: Implementation of stiff virtual walls in force-reflecting interfaces. In: Proceedings IEEE virtual reality annual international symposium (VRAIS), Seattle, WA, pp. 202-208 (1993)

26. Carmel, R., Ullrich, C., Silver, J.: VirtualHand v2.5 programmer's guide. Technical Report, Virtual Technologies Carpenter ID, Dewar RG, Ritchie JM, Simmons JEL (1996) (2001)

27. Zachmann, G., Rettig, A.: Natural and robust interaction in virtual assembly simulation. In: Proceedings 8th ISPE international conference on concurrent engineering: research and applications, Anaheim, CA (2001)

28. Zorriassatine, F., Wykes, R., Parkin, R., Gindy, N.: A survey of virtual prototyping techniques for mechanical product development. Proceedings Inst. Mech. Engineers. Part B: J. Eng. Manuf. 217(4), 513-530 (2003)

29. Garbaya, S., Coiffet, P., Blazevic, P.: Integrating computerised assembly planning with virtual assembly environment. In: Proceedings virtual reality in mechanical and production engineering (VR- Mech 2001), Brussels, Belgium (2001)

30. Dix, A., Finlay, J., Abowd, G., Beale, R.: Human-Computer Interaction, 3rd edn. PrenticeHall, Englewood Cliffs (2004) 